\title{
Research and Analysis on Intelligent Household Model based on ZigBee and Data Security Enhancement Technique
}

\author{
Wen Zhun Huang ${ }^{a^{*}}$, Yao Yao Hui ${ }^{b}$, Chao Zhang ${ }^{c}$ \\ ${ }^{1}$ Department of Electronic Information Engineering, Xijing University, Xi'an, 710123, China \\ aemail: huangwenzhun@xijing.edu.cn, bemail: 1315263876@qq.com, \\ cemail: 739275443@qq.com
}

Keywords: Intelligent Household, ZigBee, Data Security, Enhancement Technique, Analysis.

\begin{abstract}
In this paper, we conduct research on the intelligent household model based on the ZigBee and data security enhancement technique. ZigBee protocol is a kind of close, low power consumption, low cost, easy networking, high capacity, high safety standard of wireless communication technology and it is very suitable for home network of short distance wireless communication. The article design the smart home system based on ZigBee technology, complete the system framework and the overall design of ZigBee family network construction. We integrate the primary concept of the data security enhancement to optimize the traditional methods to achieve for better systematic performance.
\end{abstract}

\section{Introduction}

Smart era, electronic information enterprises for development of intelligent applications and services, content, intelligent terminal system, embedded intelligent industry related SOC chip to build for the intelligent home appliance industry, "software + chip" is the core technology, all terminal product to realize the intelligent, interconnectivity between product lineup, that formed into a strong intelligent family, that must be based on the technology platform of software and chip to achieve. Therefore, intelligent electrical appliances product development is cross-industry collaboration and multi-stage tasks, more professional team, the process of need to build corresponding intelligent home appliance development platform. With progress of information technology, large-scale intelligent information system has been in communications, military, logistics, finance business management in areas such as widely used. In the intelligent information system development process, is gradually establish the core position of data integration in the intelligent information system. To provide uniform and transparent interface, support data reuse, share and interoperability to guarantee the consistency between multiple systems promote interdisciplinary comprehensive integrated system [1-2].

Therefore, integrated data quality becomes one of the key elements of the intelligent information system effective operation. As in the whole lifecycle of the system, data quality is stable at a certain level and the data quality assessment is the basis and the necessary prerequisite to ensure the quality of the data, through the measure of data quality, estimate, validation and improvement can understand the system of data quality, and take the corresponding quality control process to improve the quality of integrated data as this is also to build intelligent information system faced one of the main topics of the quality assurance system. Smart home system as a platform to residence, integration or control all kinds of household equipment, form a set of system, structure service and general management for the integration of intelligent systems. Through local communication network are established within the residential, communications equipment and household devices can be connected to each other and as the intelligent household system basic elements within the network.

Network architecture of smart home system play a significant role in the smart home system and the wireless network with low power consumption, such as basic wireless communication transmission advantages become household system is the new trend of developing network architecture. (1) Smart home controller, the controller is responsible for a host of smart home control command to household equipment, make the equipment by executing the corresponding action to 
adjust their own state. (2) Intelligent household monitoring system as is the visual system of smart home system, the equipment status and residential environment perception, internal monitoring. (3) Intelligence lives in a database to store state information within the specified period residence, record of household equipment state information. (4) Intelligent monitoring system of residential environment is intelligence household and household equipment are an important part of cognitive monitoring, it not only assumed the job within the residential environmental data collection, responsible and even on the system level of the two-way information transmission of the smart home control host [3-4].

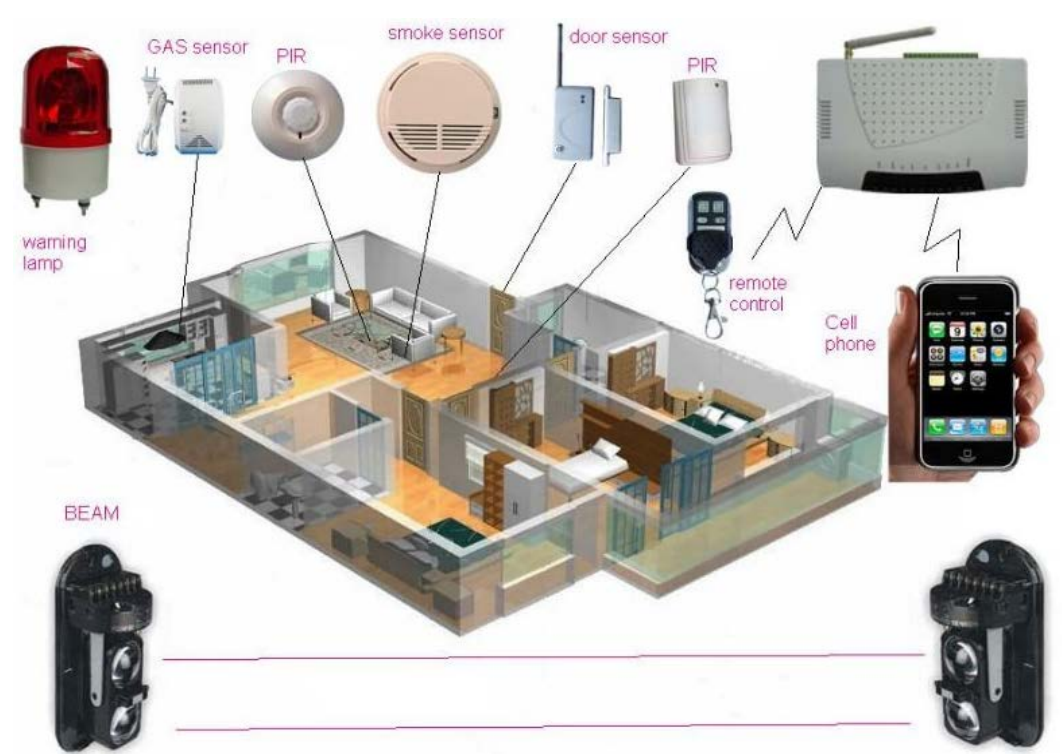

Fig. 1. The Sample Intelligent Household Model

In this article we conduct research on the intelligent household model based on the ZigBee and data security enhancement technique. As the sample, we show the demonstrative systematic description of the smart home in the figure one. In the later parts, we will introduce our method in detail.

\section{The Novel Methodology}

The Data Security Enhancement Methodologies. The network security, as the name implies, that namely focuses on the information transmission security, belongs to the important part of computer security, from the literature review, we could summarize the challenges as the follows.

- Denial of service attack. It constantly interference to network service system and change the normal process, executing independent program make the system response to slow down or even paralysis, affect the normal use of users.

- Unauthorized access. Without through approval in advance, to use the Internet or computer resources is regarded as are authorized to access, such as managed to avoid the system access control mechanism, to abnormal use of network equipment and basic resources, or its rights, unauthorized access to information [5].

- Destroy the data integrity. By illegal means that have to use the data, delete, modify, insert or resend some important information, in order to obtain benefits the attacker's response.

Symmetric cryptosystem is relatively general mature traditional encryption system to ensure the confidentiality of the data. Symmetric encryption algorithm to plaintext and key encrypted algorithm together, send encrypted form ciphertext with the decryption use the encrypted key and encryption algorithm of the inverse algorithm of cipher decryption, restore clear information. Its advantages are simple algorithm and publicly, fast encryption speed, small amount of the calculation, high efficiency, operation occupy less resources, at present is still one of the mainstream of the cryptosystem. Cloud computing environment compared to the previous technology as large number of using virtualization technology, how to solve the security at the aspect of virtualization and cloud computing security and thee traditional security and a significant difference. 
Under this background, we propose the listed suggestions for optimization. (1) A firewall. Firewall provide network address translation functions, to ensure that the Intranet subnet and host local subnet address is not exposed to the public network to protect the subnet information resources, at the same time as the subnet host access to the Internet to provide public network equipment can recognize global IP address as the IP packet filtering according to the system management of the security policy configuration. (2) Virtual private network. VPN implementation in TCP/IP environment information security exchange according to the configuration of system management requirements, to establish safety connection between the firewall, implementation of confidential data encryption transmission, ensure confidentiality and integrity of information through public. Through VPN IPsec protocol and tunnel protocol can achieve much connection. Through a series of means of encryption, the VPN can become like a real dedicated security as demonstrated in the Figure 2.

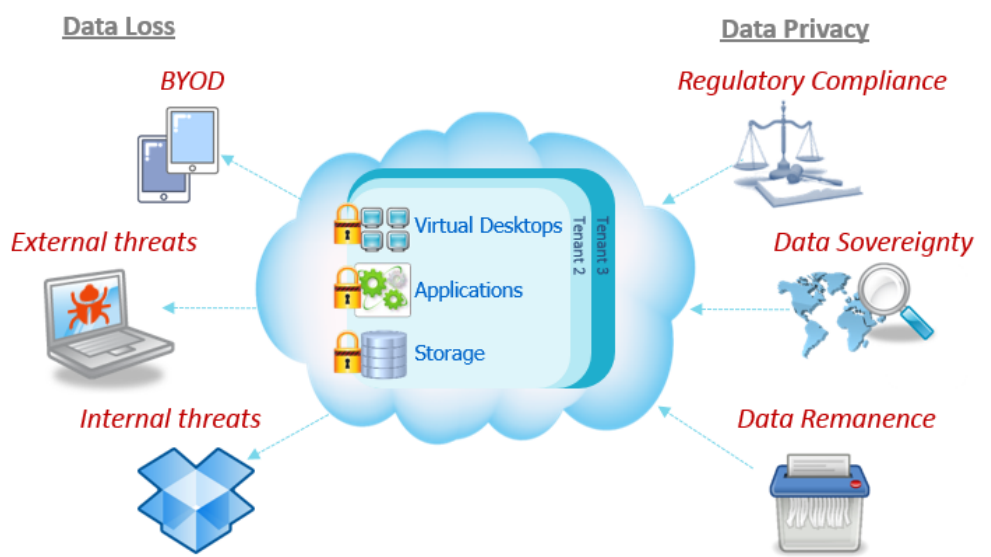

Fig. 2. The Data Security Enhancement Countermeasures

The ZigBee and Smart Home. ZigBee protocol is by the ZigBee alliance with cheap, low power consumption, the two-way communication as the goal of a wireless communication protocol, mainly is embedded in a variety of electronic equipment, household equipment and building automation and the industrial control field, etc. Smart home system using ZigBee agreement, can be composed of the simple structure, flexible, throughput, limited power of local area network within the wireless local area network equipment is divided into a fully functional and reduced function devices. The former as the coordinator, routers and network form is responsible for the smart home system and smart home system in the intelligent monitoring system and data exchange between host smart home control as the latter can only be as intelligent terminal nodes, the state of the residential interior equipment data and environmental parameters that not down for the extension of the network [6-7].

With the ZigBee, we could suggest efficient smart home architecture as follows. (1) Coordinator is the core of the whole system network center, is responsible for establishing network and management network nodes, forming a ZigBee network. Through the ZigBee network is able to communicate with a terminal node that will be received by the home gateway control command routing and passed to the terminal node and can also be of basic sensor data acquisition terminal node, and analyzing the data processing. (2) Terminal node is composed of multiple sensor nodes of wireless sensor networks, the data source is intelligence household environment, is also the concrete executor of control commands. (3) Home gateway is the core of the internal network and external network for information exchange and it is mainly responsible for protocol conversion and transmission of the information. The user can through the mobile communication network, the exchange of information, Internet and smart home system for real-time monitoring information in the home.

The transport layer is based on the characteristics of communication subnet, the best use of network resources, for the two end systems between the session layer, providing connection to establish, with maintain and cancel the transfer function, control the flow of data transmission, ensure the quality of basic communication services as the application layer is at the top of the open system, directly provide services for the application process, including a series of the application layer 
software based on the monitoring task. Wireless sensor network in the family in we choose the basic full-featured network coordinator for use as a control point of home appliance, and instruction in this point to transform the network in order to convert from outside the network instruction to their instructions to control the nodes in the network. In the figure three, we show the sample architecture.

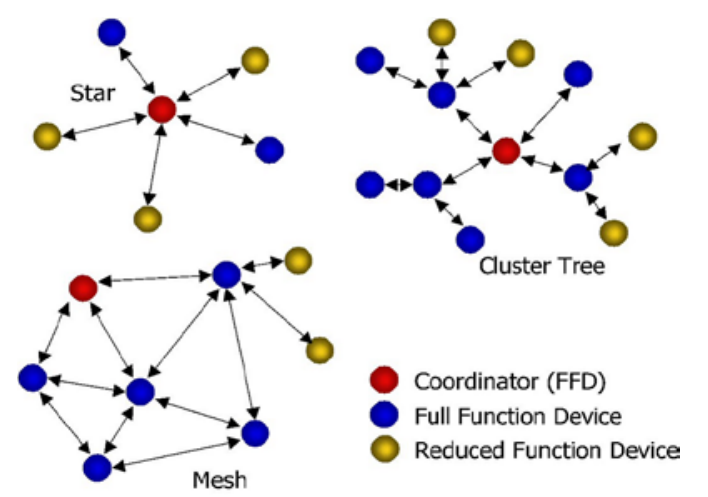

Fig. 3. The ZigBee Topology Distribution Pattern

The Data Assisted Smart Home. Big data intelligent analysis need new theory and technology of the breakthrough, but it with the traditional intelligent data analysis methods must also continue. The traditional data analysis field, such as knowledge base system, data warehouse and data mining theory and technology has accumulated a lot of good and effective algorithms.

Any external devices can receive network devices to send data at the same time can also send data to the equipment, so the network is very vulnerable to the outside world. At the same time, intelligent household data transmission to ensure the general integrity, confidentiality, so the security of the data transmission in intelligent home network research is extremely important. The realization of system task by two different processes: the local data acquisition program and network services. (1) Network service program is actually made up of the two parts: the embedded Webserver and CGI programs. Embedded Web server as daemon runs in the background that is responsible for monitoring customer requests from the network. When the user request to the local system through basic GSM network communication, start the corresponding CGI program, the request into the server can recognize basic format, after processing, again by CGI convert the results into a web browser can recognize format, as a reply message back to the client, so as to complete the client interactions with local system. (2) The local data collection procedures according to the task are divided into following modules: data collection module, data processing module, LCD module, data storage module, GSM communication module. Data acquisition module is responsible for the control parameters set by the user, the external signal acquisition, and collected data to the data processing module [8].

The Enhanced Smart Home Architecture. Through the above introduction to technical and the functional requirements analysis, combined with the existing design, this paper gives the smart home control system mainly includes the central management unit, external communication and the internal communication of three parts.

- Internal communications: inside the smart home system, adopt the way of ZigBee network between each node, with Mesh network in network topology method or the combination of the basic tree network.

- External communications: external via WIFI is connected with the central management unit, used to send control instructions, etc.

- Because of the complexity of the actual operation and cost issues, this article put forward after the home control system with the method of the experiment to verify, that can be achieved by using the method of this scheme of the portable remote control and the PC to simulation on handheld devices, access via TCP/IP mode that can complete in the future development of the software on handheld devices.

Smart home can connect a wide range of the devices in the home through the Internet of things technology together to provide home appliances control, lighting control, telephone remote control, environmental monitoring, indoor and outdoor, infrared remote control, anti-theft alarm and control 
forward and programmable timing control and other functions and means, can provide a full range of information interaction function, and can save energy and money that can be reflected from the listed aspects. (1) Send corresponding instructions through the upper machine, various forms of the wireless control to high precision stepper motor rotation, so as to realize the automatic switch to the door, the electric curtain control. (2) Send corresponding instructions through the upper machine, wireless control temperature and humidity data acquisition node temperature and humidity, and then the data back to the PC and realize environmental monitoring function. (3) Send corresponding instructions through the upper machine, wireless control relay switch state, to achieve their work in the big voltage or current electrical wireless on or off, namely, home appliance control and other functions.

\section{Summary and Conclusion}

In this paper, we conduct research on the intelligent household model based on the ZigBee and data security enhancement technique. The development of the intelligent device has been used in many fields. In today's information technology and the rapid development of general modern science and technology, people pay more attention to is in achieving the required functions of how to reduce the power consumption at the same time, the more convenient and more humanized control equipment, looking for a better man-machine interaction. The key technology of the smart home system is build family internal network and intelligent control. Wireless network technology of flexible installation and the characteristics of mobile makes it more and more been used in intelligent system. This paper designs a wireless intelligent household system based on ZigBee technology has the close, low power consumption, low cost, easy networking, high capacity and high security advantages. In the further research, we will implement the system and test the feasibility.

\section{Acknowledgements}

In this paper, the research was sponsored by the 2015 Training Project of National College Students Innovation and entrepreneurship (Project No. 127152015010).

\section{References}

[1] Marques, Ion, et al. An experiment of subconscious intelligent social computing on household appliances. Neurocomputing 167 (2015): 32-43.

[2] Yan, Gui Lin, et al. The Design of the Household Photovoltaic Power-Supply System with Intelligent Expansion Capacity Based on STM32. Advanced Materials Research. Vol. 1044. Trans Tech Publications, 2014.

[3] QU, Mingfei, and Dan ZHAO. Intelligent Shutter System Design of Photovoltaic Power Generation Based on Single Chip Microcomputer Control. (2015).

[4] Liu, Lanbin, Lin Fu, and Yi Jiang. A new “wireless on-off control” technique for adjusting and metering household heat in district heating system. Applied Thermal Engineering 36 (2012): 202-209.

[5] Lu, Ning, et al. A multi-layer, data-driven advanced reasoning tool for intelligent data mining and analysis for smart grids. Power and Energy Society General Meeting, 2012 IEEE. IEEE, 2012.

[6] Aziz, A. F. A., et al. Artificial intelligent meter development based on advanced metering infrastructure technology. Renewable and Sustainable Energy Reviews 27 (2013): 191-197.

[7] ZHANG, Jie, Hui-bin QIN, and Xiang-gen MAO. Design of indoor intelligent lighting systems based on ZigBee. Internet of Things Technologies 9 (2013): 014. 
[8] Zhen-Hai, M. U. The Design and Implementation of Intelligent Office Systems based on the Intelligent Interactive Technology. Academic Journal of Manufacturing Engineering 12.1 (2014). 